Supporting Information for

Structural Characterization of Mn(II) Compounds with Methylalumoxane (MAO) and $\mathrm{AlMe}_{2}$ unit.

Lucjan B. Jerzykiewicz, Józef Utko, and Piotr Sobota*

$$
\begin{aligned}
& \text { Faculty of Chemistry, } \\
& \text { University of Wrocław, } \\
& \text { 14. F. Joliot Curie, } \\
& \text { 50-383 Wrocław, } \\
& \text { Poland }
\end{aligned}
$$




\section{Notation and formulas}

1

$\left[\mathrm{Mn}_{4}(\mu-\mathrm{Cl}) \mathrm{Cl}_{3}\left(\mathrm{OCH}_{2} \mathrm{CH}_{2} \mathrm{OCH}_{3}\right)_{4}\left(\mathrm{HOCH}_{2} \mathrm{CH}_{2} \mathrm{OCH}_{3}\right)_{3}\right]_{2}$

$\mathrm{C}_{42} \mathrm{H}_{104} \mathrm{Cl}_{8} \mathrm{Mn}_{8} \mathrm{O}_{28}$

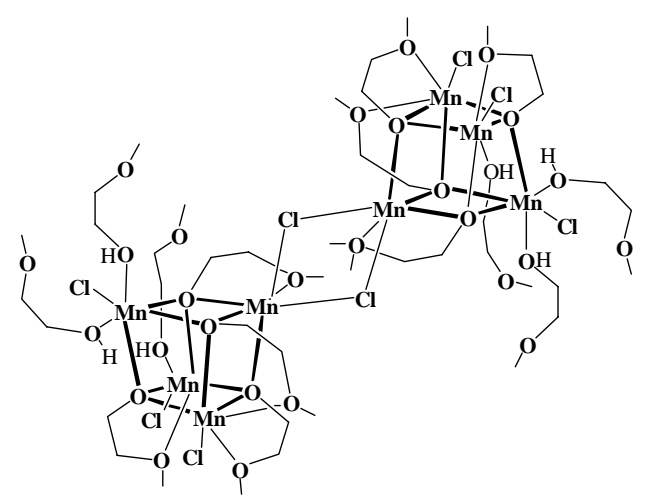

2

$\left.\left[\mathrm{Mn}_{3} \mathrm{Al}\left(\mu_{3}-\mathrm{OCH}_{2} \mathrm{CH}_{2} \mathrm{OCH} \mathrm{H}_{3}\right)(\mu-\mathrm{Cl})_{3}\left(\mu-\mathrm{OCH}_{2} \mathrm{CH}_{2} \mathrm{OCH}_{3}\right)_{2} \text { (thf }\right)_{2}\left(\mathrm{CH}_{3}\right)_{2} \mathrm{Cl}\right]$

$\mathrm{C}_{19} \mathrm{H}_{43} \mathrm{AlCl}_{4} \mathrm{Mn}_{3} \mathrm{O}_{8}$

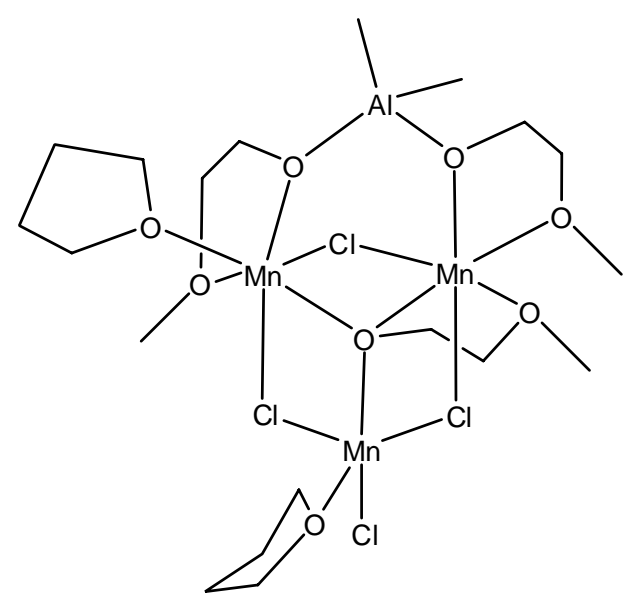

3

$\left[\mathrm{MnAl}_{3}\left(\mu_{3}-\mathrm{O}\right)\left(\mu-\mathrm{OCH}_{2} \mathrm{CH}_{2} \mathrm{OCH}_{3}\right)_{3}\left(\mathrm{CH}_{3}\right)_{6}\right]$

$\mathrm{C}_{19} \mathrm{H}_{43} \mathrm{AlCl}{ }_{4} \mathrm{Mn}_{3} \mathrm{O}_{8}$

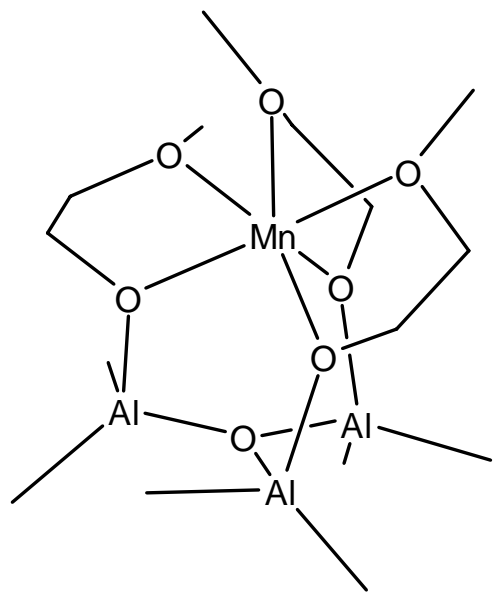




\section{Experimental Section}

Preparation of 1 .

General: All reactions were conducted under $\mathrm{N}_{2}$ atmospheres. Chemicals were treated as follows: thf, distilled from $\mathrm{CuCl}$, predried over $\mathrm{NaOH}$, and then distilled from Na/benzophenone; toluene, distilled from Na/benzophenone; hexanes, distilled from $\mathrm{P}_{2} \mathrm{O}_{5} ; \mathrm{CH}_{3} \mathrm{CN}$, distilled from $\mathrm{CaH}_{2}$, and 2-methoxyethanol (Aldrich) distilled prior to use. Metallic $\mathrm{Mn}$ and $\mathrm{NH}_{4} \mathrm{Cl}$ (Aldrich) used as received.

Microanalyses were conducted by ASA-1 (GDR, Karl-Zeiss-Jena) instrument (in-house).

\section{$\left[\mathrm{Mn}_{4}(\boldsymbol{\mu}-\mathbf{C l}) \mathrm{Cl}_{3}\left(\mathbf{O C H}_{2} \mathbf{C H}_{2} \mathbf{O C H}\right)_{4}\left(\mathbf{H O C H}_{2} \mathbf{C H}_{2} \mathbf{O} \mathrm{CH}_{3}\right)_{3}\right]_{2},{ }^{1}$ (1): Manganese metal $(2.20 \mathrm{~g}$,} $40.04 \mathrm{mmol}$ ) was added to a suspension of $\mathrm{NH}_{4} \mathrm{Cl}$ (2.14 g, $40.04 \mathrm{mmol}$ ) in a mixture of $\mathrm{CH}_{3} \mathrm{OCH}_{2} \mathrm{CH}_{2} \mathrm{OH}$ (32 $\left.\mathrm{mL}\right)$ and toluene $(40 \mathrm{~mL})$. Evolution of $\mathrm{HCl}$ and $\mathrm{H}_{2}$ started immediately. The mixture was stirred under reflux until all metal was consumed (usually a week). The mixture was cooled down to room temperature, filtered and the volatiles were removed under vacuum. $100 \mathrm{~cm}^{3}$ of hexanes were added to the resulted oily powder and the mixture was stirred, filtered off washed with hexanes $\left(3 \times 15 \mathrm{~cm}^{3}\right.$ ) and dried to give $\mathbf{1}$ as a brown powder (7.78g, 87.4 ․). Elemental analysis calcd for $\mathrm{C}_{42} \mathrm{H}_{104} \mathrm{Cl}_{8} \mathrm{Mn}_{8} \mathrm{O}_{28}$ : C 28.33, $\mathrm{H}$ 5.89, Cl 15.93; found: C $28.01, \mathrm{H} 5.72, \mathrm{Cl} 19.75$.

\footnotetext{
I Jerzykiewicz, L. J., Utko, J., Duczmal, M., Sobota, P., Submitted to Chem.Commun.
} 


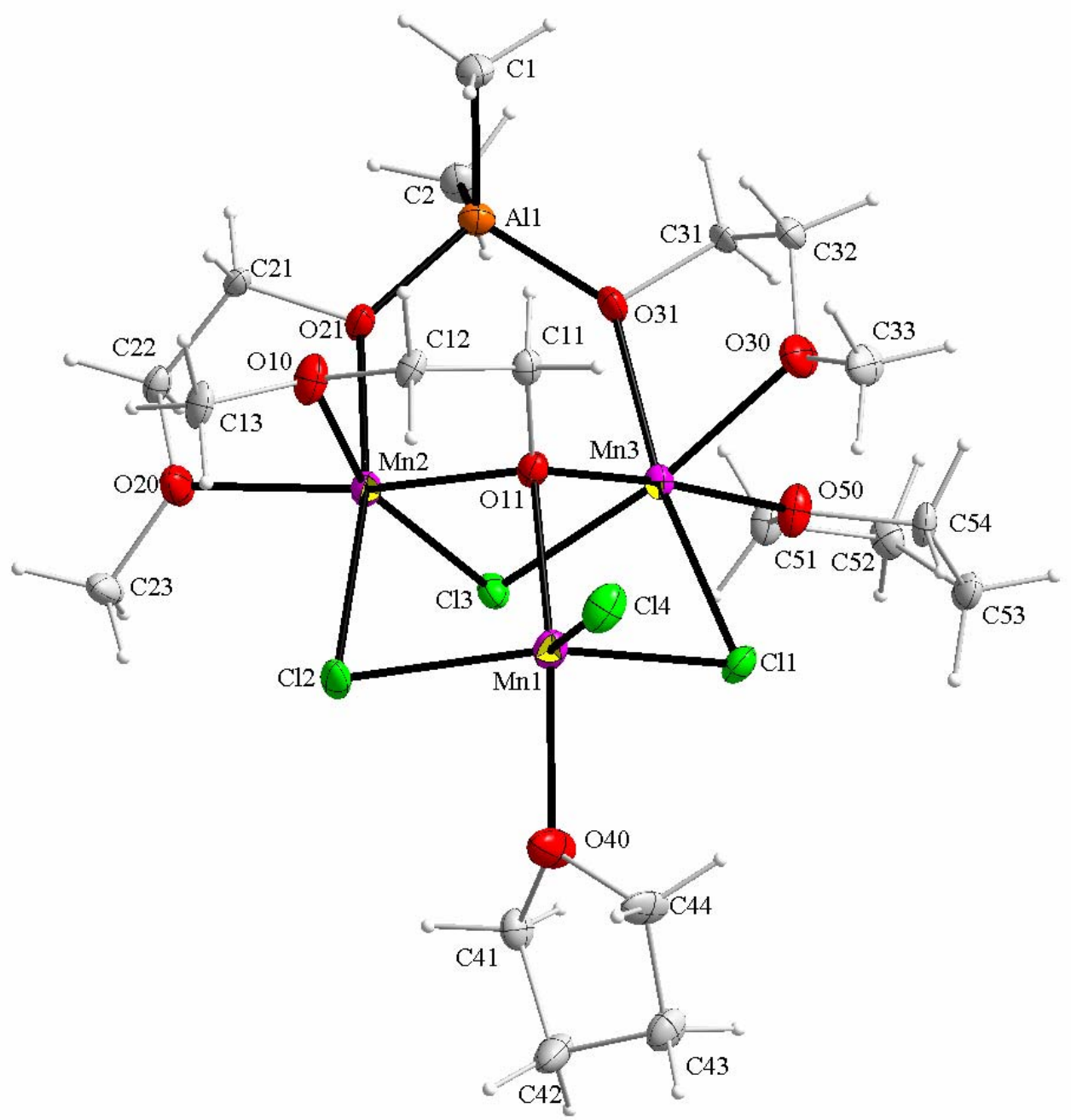

Figure S1. Molecular structure of

the $\left[\mathrm{Mn}{ }_{3} \mathrm{Al}\left(\mu_{3}-\mathrm{OCH}_{2} \mathrm{CH}_{2} \mathrm{OCH}\right)(\mu-\mathrm{Cl})_{3}\left(\mu-\mathrm{OCH}_{2} \mathrm{CH}_{2} \mathrm{OCH}_{3}\right)_{2}(\text { thf })_{2}(\mathrm{Me})_{2} \mathrm{Cl}\right]$ (ORTEP plot, thermal ellipsoids set at 50 \%). 
Table S11. Crystal data and structure refinement for

\section{$\left[\mathrm{Mn}_{3} \mathrm{Al}\left(\mathrm{\mu}_{3}-\mathrm{OCH}_{2} \mathrm{CH}_{2} \mathrm{OCH}_{3}\right)(\mu-\mathrm{Cl})_{3}\left(\mu-\mathrm{OCH}_{2} \mathrm{CH}_{2} \mathrm{OCH}_{3}\right)_{2}(\text { thf })_{2}(\mathrm{Me})_{2} \mathrm{Cl}\right]$ (2).}

\section{Compound}

Chemical formula

Formula weight

$\lambda(\AA)$

$\mathrm{T}(\mathrm{K})$

Space group

Crystal system

Unit cell dimensions

a $(\AA)$

$\mathrm{b}(\AA)$

$c(\AA)$

alpha $\left({ }^{\circ}\right)$

beta $\left({ }^{\circ}\right)$

gamma $\left({ }^{\circ}\right)$

$\mathrm{V}\left(\AA^{3}\right)$

Z

$\mathrm{D}_{\mathrm{C}}\left(\mathrm{Mg} / \mathrm{m}^{3}\right)$

$\mathrm{F}(000)$

Habit

Crystal size (mm)

$\mu\left(\mathrm{mm}^{-1}\right)$

Absorption correction

Max. and min.

Type of diffractometer

Diffraction geometry

Theta range $\left({ }^{\circ}\right)$

Number of reflections measured

Number of unique reflections

$\mathrm{R}_{\text {(int) }}$

Number of observed reflections

Refinement method

Number of parameters

Final R1 indices [I>2 $\sigma(I)]$

Final R1 indices (all data)

Goodness-of-fit (S)

Largest diff. peak and hole e. $\AA^{-3}$
CCDC299461

$\mathrm{C}_{19} \mathrm{H}_{43} \mathrm{AlCl}_{4} \mathrm{Mn}_{3} \mathrm{O}_{8}$

733.13

0.71073

$100(2)$

$\mathrm{P} 21 / \mathrm{n}$

Monoclinic

$14.271(4)$

$15.302(5)$

$14.920(5)$

90

$91.69(2)$

90

3256.7 ( 18$)$

4

1.495

1508

block

$0.293 \times 0.264 \times 0.200$

1.536

Analytical

transmission 0.910 and 0.687

Kuma KM4CCD kappa-axis diffractometer $\omega$

2.99 to 27.00

21678

7023

0.0541

$5119[\mathrm{I}>2 \sigma(\mathrm{I})]$

least-squares on $\mathrm{F}^{2}$

321

$\mathrm{R}_{1}=0.0442, \mathrm{wR}_{2}=0.0968$

$\mathrm{R}_{1}=0.0680, \mathrm{wR}_{2}=0.1027$

1.012

0.670 and -0.668 
Table S12. Bond lengths [A] and angles [deg] for $\left[\mathrm{Mn}_{3} \mathrm{Al}\left(\mu_{3}-\mathrm{OCH}_{2} \mathrm{CH}_{2} \mathrm{OCH}_{3}\right)(\mu-\mathrm{Cl})_{3}\left(\mu-\mathrm{OCH}_{2} \mathrm{CH}_{2} \mathrm{OCH}_{3}\right)_{2}(\mathrm{thf})_{2}(\mathrm{Me})_{2} \mathrm{Cl}\right]$

\begin{tabular}{|c|c|}
\hline $\operatorname{Mn}(1)-O(11)$ & $2.186(2)$ \\
\hline $\operatorname{Mn}(1)-O(40)$ & $2.202(2)$ \\
\hline $\operatorname{Mn}(1)-\mathrm{Cl}(4)$ & $2.334(2)$ \\
\hline $\operatorname{Mn}(1)-\mathrm{Cl}(1)$ & $2.474(2)$ \\
\hline $\operatorname{Mn}(1)-\mathrm{Cl}(2)$ & $2.498(2)$ \\
\hline $\operatorname{Mn}(2)-O(21)$ & $2.138(2)$ \\
\hline $\operatorname{Mn}(2)-O(11)$ & $2.160(2)$ \\
\hline $\operatorname{Mn}(2)-0(20)$ & $2.218(2)$ \\
\hline $\operatorname{Mn}(2)-O(10)$ & $2.292(2)$ \\
\hline $\operatorname{Mn}(2)-\operatorname{Cl}(2)$ & $2.490(2)$ \\
\hline $\operatorname{Mn}(2)-\mathrm{Cl}(3)$ & $2.503(2)$ \\
\hline $\operatorname{Mn}(3)-O(31)$ & $2.151(2)$ \\
\hline $\operatorname{Mn}(3)-O(11)$ & $2.167(2)$ \\
\hline $\operatorname{Mn}(3)-0(50)$ & $2.187(2)$ \\
\hline $\operatorname{Mn}(3)-O(30)$ & $2.255(2)$ \\
\hline $\operatorname{Mn}(3)-\mathrm{Cl}(3)$ & $2.493(1)$ \\
\hline $\operatorname{Mn}(3)-\mathrm{Cl}(1)$ & $2.532(2)$ \\
\hline$A 1(1)-O(21)$ & $1.801(2)$ \\
\hline$A 1(1)-O(31)$ & $1.806(2)$ \\
\hline $\mathrm{Al}(1)-\mathrm{C}(1)$ & $1.972(3)$ \\
\hline $\mathrm{Al}(1)-\mathrm{C}(2)$ & $1.972(3)$ \\
\hline$O(10)-C(13)$ & $1.443(4)$ \\
\hline $\mathrm{O}(10)-\mathrm{C}(12)$ & $1.446(4)$ \\
\hline $\mathrm{O}(11)-\mathrm{C}(11)$ & $1.436(3)$ \\
\hline $\mathrm{O}(20)-\mathrm{C}(23)$ & $1.440(4)$ \\
\hline $\mathrm{O}(20)-\mathrm{C}(22)$ & $1.450(4)$ \\
\hline $\mathrm{O}(21)-\mathrm{C}(21)$ & $1.432(4)$ \\
\hline $\mathrm{O}(30)-\mathrm{C}(33)$ & $1.429(4)$ \\
\hline $\mathrm{O}(30)-\mathrm{C}(32)$ & $1.439(4)$ \\
\hline $\mathrm{O}(31)-\mathrm{C}(31)$ & $1.439(4)$ \\
\hline $\mathrm{O}(40)-\mathrm{C}(41)$ & $1.448(4)$ \\
\hline $\mathrm{O}(40)-\mathrm{C}(44)$ & $1.460(4)$ \\
\hline$O(50)-C(54)$ & $1.455(4)$ \\
\hline $\mathrm{O}(50)-\mathrm{C}(51)$ & $1.458(4)$ \\
\hline$C(31)-C(32)$ & $1.507(5)$ \\
\hline$C(11)-C(12)$ & $1.504(4)$ \\
\hline$C(21)-C(22)$ & $1.505(4)$ \\
\hline$C(51)-C(52)$ & $1.512(5)$ \\
\hline$C(52)-C(53)$ & $1.528(5)$ \\
\hline$C(53)-C(54)$ & $1.502(5)$ \\
\hline$C(41)-C(42)$ & $1.507(5)$ \\
\hline$C(42)-C(43)$ & $1.498(5)$ \\
\hline$C(43)-C(44)$ & $1.491(5)$ \\
\hline$O(11)-\operatorname{Mn}(1)-O(40)$ & $159.55(8)$ \\
\hline $\mathrm{O}(11)-\mathrm{Mn}(1)-\mathrm{Cl}(4)$ & $104.39(6)$ \\
\hline $\mathrm{O}(40)-\mathrm{Mn}(1)-\mathrm{Cl}(4)$ & $96.06(7)$ \\
\hline $\mathrm{O}(11)-\mathrm{Mn}(1)-\mathrm{Cl}(1)$ & $84.87(6)$ \\
\hline $\mathrm{O}(40)-\mathrm{Mn}(1)-\mathrm{Cl}(1)$ & $88.66(7)$ \\
\hline $\mathrm{Cl}(4)-\mathrm{Mn}(1)-\mathrm{Cl}(1)$ & $108.08(4)$ \\
\hline $\mathrm{O}(11)-\mathrm{Mn}(1)-\mathrm{Cl}(2)$ & $84.97(6)$ \\
\hline $\mathrm{O}(40)-\mathrm{Mn}(1)-\mathrm{Cl}(2)$ & $84.14(6)$ \\
\hline $\mathrm{Cl}(4)-\mathrm{Mn}(1)-\mathrm{Cl}$ (2) & $122.72(4)$ \\
\hline $\mathrm{Cl}(1)-\mathrm{Mn}(1)-\mathrm{Cl}(2)$ & $129.15(4)$ \\
\hline
\end{tabular}




\begin{tabular}{|c|c|}
\hline$O(21)-\operatorname{Mn}(2)-O(11)$ & $102.16(8)$ \\
\hline$O(21)-\operatorname{Mn}(2)-O(20)$ & $77.37(8)$ \\
\hline$O(11)-\operatorname{Mn}(2)-O(20)$ & $166.53(8)$ \\
\hline$O(21)-\operatorname{Mn}(2)-O(10)$ & $87.12(8)$ \\
\hline$O(11)-\operatorname{Mn}(2)-O(10)$ & $76.08(8)$ \\
\hline$O(20)-\operatorname{Mn}(2)-O(10)$ & $90.46(8)$ \\
\hline $\mathrm{O}(21)-\mathrm{Mn}(2)-\mathrm{Cl}(2)$ & $170.20(6)$ \\
\hline $\mathrm{O}(11)-\mathrm{Mn}(2)-\mathrm{Cl}(2)$ & $85.71(6)$ \\
\hline $\mathrm{O}(20)-\mathrm{Mn}(2)-\mathrm{Cl}(2)$ & $93.60(6)$ \\
\hline $\mathrm{O}(10)-\mathrm{Mn}(2)-\mathrm{Cl}(2)$ & $89.16(6)$ \\
\hline$O(21)-\operatorname{Mn}(2)-\operatorname{Cl}(3)$ & $92.64(6)$ \\
\hline $\mathrm{O}(11)-\mathrm{Mn}(2)-\mathrm{Cl}(3)$ & $85.76(6)$ \\
\hline$O(20)-\operatorname{Mn}(2)-C l(3)$ & $107.71(6)$ \\
\hline$O(10)-\operatorname{Mn}(2)-\mathrm{Cl}(3)$ & $161.34(6)$ \\
\hline $\mathrm{Cl}(2)-\mathrm{Mn}(2)-\mathrm{Cl}(3)$ & $93.77(3)$ \\
\hline$O(31)-\mathrm{Mn}(3)-O(11)$ & $93.49(8)$ \\
\hline$O(31)-\operatorname{Mn}(3)-O(50)$ & $92.64(8)$ \\
\hline$O(11)-\operatorname{Mn}(3)-O(50)$ & $173.61(8)$ \\
\hline$O(31)-\operatorname{Mn}(3)-O(30)$ & $75.22(8)$ \\
\hline$O(11)-\operatorname{Mn}(3)-O(30)$ & $95.74(8)$ \\
\hline$O(50)-\operatorname{Mn}(3)-O(30)$ & $84.11(9)$ \\
\hline$O(31)-\operatorname{Mn}(3)-C 1(3)$ & $95.34(6)$ \\
\hline$O(11)-\operatorname{Mn}(3)-\mathrm{Cl}(3)$ & $85.88(6)$ \\
\hline$O(50)-\operatorname{Mn}(3)-C l(3)$ & $95.32(6)$ \\
\hline$O(30)-\operatorname{Mn}(3)-C l(3)$ & $170.48(6)$ \\
\hline $\mathrm{O}(31)-\mathrm{Mn}(3)-\mathrm{Cl}(1)$ & $167.73(6)$ \\
\hline $\mathrm{O}(11)-\mathrm{Mn}(3)-\mathrm{Cl}(1)$ & $83.88(6)$ \\
\hline$O(50)-\mathrm{Mn}(3)-\mathrm{Cl}(1)$ & $89.75(7)$ \\
\hline $\mathrm{O}(30)-\mathrm{Mn}(3)-\mathrm{Cl}(1)$ & $93.08(6)$ \\
\hline $\mathrm{Cl}(3)-\mathrm{Mn}(3)-\mathrm{Cl}(1)$ & $96.43(3)$ \\
\hline $\operatorname{Mn}(1)-\mathrm{Cl}(1)-\operatorname{Mn}(3)$ & $85.20(3)$ \\
\hline $\operatorname{Mn}(2)-\operatorname{Cl}(2)-\operatorname{Mn}(1)$ & $85.12(3)$ \\
\hline $\operatorname{Mn}(3)-C l(3)-\operatorname{Mn}(2)$ & $81.92(4)$ \\
\hline$O(21)-A 1(1)-O(31)$ & $102.11(10)$ \\
\hline$O(21)-A l(1)-C(1)$ & $110.11(13)$ \\
\hline$O(31)-A I(1)-C(1)$ & $109.69(14)$ \\
\hline$O(21)-A I(1)-C(2)$ & $111.01(14)$ \\
\hline$O(31)-A I(1)-C(2)$ & $111.22(14)$ \\
\hline$C(1)-A 1(1)-C(2)$ & $112.25(16)$ \\
\hline$C(13)-O(10)-C(12)$ & $111.3(2)$ \\
\hline$C(13)-O(10)-\operatorname{Mn}(2)$ & $120.57(19)$ \\
\hline $\mathrm{C}(12)-\mathrm{O}(10)-\mathrm{Mn}(2)$ & $111.09(17)$ \\
\hline $\mathrm{C}(11)-\mathrm{O}(11)-\mathrm{Mn}(2)$ & $109.93(17)$ \\
\hline $\mathrm{C}(11)-\mathrm{O}(11)-\mathrm{Mn}(3)$ & $122.82(17)$ \\
\hline $\operatorname{Mn}(2)-O(11)-\operatorname{Mn}(3)$ & $98.36(8)$ \\
\hline $\mathrm{C}(11)-\mathrm{O}(11)-\mathrm{Mn}(1)$ & $118.16(17)$ \\
\hline $\operatorname{Mn}(2)-O(11)-\operatorname{Mn}(1)$ & $101.83(8)$ \\
\hline $\operatorname{Mn}(3)-O(11)-\operatorname{Mn}(1)$ & $102.22(8)$ \\
\hline$C(23)-O(20)-C(22)$ & $112.5(2)$ \\
\hline$C(23)-O(20)-\operatorname{Mn}(2)$ & $126.2(2)$ \\
\hline $\mathrm{C}(22)-\mathrm{O}(20)-\mathrm{Mn}(2)$ & $111.34(18)$ \\
\hline$C(21)-O(21)-A 1(1)$ & $118.20(18)$ \\
\hline $\mathrm{C}(21)-\mathrm{O}(21)-\mathrm{Mn}(2)$ & $108.65(17)$ \\
\hline $\operatorname{Al}(1)-O(21)-\operatorname{Mn}(2)$ & $132.05(11)$ \\
\hline$C(33)-O(30)-C(32)$ & $113.0(2)$ \\
\hline$C(33)-O(30)-\operatorname{Mn}(3)$ & $130.1(2)$ \\
\hline $\mathrm{C}(32)-\mathrm{O}(30)-\mathrm{Mn}(3)$ & $113.30(18)$ \\
\hline$C(31)-O(31)-A 1(1)$ & $114.91(19)$ \\
\hline $\mathrm{C}(31)-\mathrm{O}(31)-\mathrm{Mn}(3)$ & $107.46(18)$ \\
\hline $\operatorname{Al}(1)-O(31)-\operatorname{Mn}(3)$ & $137.43(11)$ \\
\hline$C(41)-O(40)-C(44)$ & $108.3(3)$ \\
\hline
\end{tabular}




\begin{tabular}{|c|c|}
\hline $\mathrm{C}(41)-\mathrm{O}(40)-\mathrm{Mn}(1)$ & $122.79(19)$ \\
\hline $\mathrm{C}(44)-\mathrm{O}(40)-\mathrm{Mn}(1)$ & $120.2(2)$ \\
\hline$C(54)-O(50)-C(51)$ & $109.4(2)$ \\
\hline $\mathrm{C}(54)-\mathrm{O}(50)-\mathrm{Mn}(3)$ & $126.40(18)$ \\
\hline$C(51)-O(50)-\operatorname{Mn}(3)$ & $124.14(18)$ \\
\hline $\mathrm{O}(31)-\mathrm{C}(31)-\mathrm{C}(32)$ & $109.4(3)$ \\
\hline $\mathrm{O}(30)-\mathrm{C}(32)-\mathrm{C}(31)$ & $107.5(3)$ \\
\hline $\mathrm{O}(11)-\mathrm{C}(11)-\mathrm{C}(12)$ & $110.6(3)$ \\
\hline $\mathrm{O}(21)-\mathrm{C}(21)-\mathrm{C}(22)$ & $109.6(2)$ \\
\hline $\mathrm{O}(20)-\mathrm{C}(22)-\mathrm{C}(21)$ & $106.6(2)$ \\
\hline$O(50)-C(51)-C(52)$ & $105.9(3)$ \\
\hline$C(51)-C(52)-C(53)$ & $102.8(3)$ \\
\hline$C(54)-C(53)-C(52)$ & $102.2(3)$ \\
\hline$O(50)-C(54)-C(53)$ & $104.6(3)$ \\
\hline $\mathrm{O}(40)-\mathrm{C}(41)-\mathrm{C}(42)$ & $104.6(3)$ \\
\hline$C(43)-C(42)-C(41)$ & $103.6(3)$ \\
\hline$C(44)-C(43)-C(42)$ & $103.4(3)$ \\
\hline$O(40)-C(44)-C(43)$ & $107.4(3)$ \\
\hline
\end{tabular}


Table S13. Torsion angles [deg] for

$\left[\mathrm{Mn}{ }_{3} \mathrm{Al}\left(\mu_{3}-\mathrm{OCH}_{2} \mathrm{CH}_{2} \mathrm{OCH}_{3}\right)(\mu-\mathrm{Cl})_{3}\left(\mu-\mathrm{OCH}_{2} \mathrm{CH}_{2} \mathrm{OCH}_{3}\right)_{2}(\mathrm{thf})_{2}(\mathrm{Me})_{2} \mathrm{Cl}\right]$ (2).

\begin{tabular}{|c|c|}
\hline $\mathrm{Cl}(2)-\mathrm{Mn}(1)-\mathrm{Cl}(1)-\mathrm{Mn}(3)$ & $65.72(5)$ \\
\hline $\mathrm{Cl}(4)-\mathrm{Mn}(1)-\mathrm{Cl}(1)-\mathrm{Mn}(3)$ & $-117.01(4)$ \\
\hline $\mathrm{O}(11)-\mathrm{Mn}(1)-\mathrm{Cl}(1)-\mathrm{Mn}(3)$ & $-13.53(6)$ \\
\hline$O(40)-\operatorname{Mn}(1)-\mathrm{Cl}(1)-\mathrm{Mn}(3)$ & $147.06(7)$ \\
\hline $\mathrm{Cl}(1)-\mathrm{Mn}(1)-\mathrm{Cl}(2)-\mathrm{Mn}(2)$ & $-68.53(5)$ \\
\hline $\mathrm{Cl}(4)-\mathrm{Mn}(1)-\mathrm{Cl}(2)-\mathrm{Mn}(2)$ & $114.56(4)$ \\
\hline$O(11)-\operatorname{Mn}(1)-\mathrm{Cl}(2)-\mathrm{Mn}(2)$ & $10.68(6)$ \\
\hline $\mathrm{O}(40)-\mathrm{Mn}(1)-\mathrm{Cl}(2)-\mathrm{Mn}(2)$ & $-151.99(7)$ \\
\hline $\mathrm{Cl}(1)-\mathrm{Mn}(1)-\mathrm{O}(11)-\mathrm{Mn}(2)$ & $117.55(7)$ \\
\hline $\mathrm{Cl}(1)-\mathrm{Mn}(1)-\mathrm{O}(11)-\mathrm{Mn}(3)$ & $16.18(7)$ \\
\hline $\mathrm{Cl}(1)-\mathrm{Mn}(1)-\mathrm{O}(11)-\mathrm{C}(11)$ & $-121.95(18)$ \\
\hline $\mathrm{Cl}(2)-\mathrm{Mn}(1)-\mathrm{O}(11)-\mathrm{Mn}(2)$ & $-12.56(7)$ \\
\hline $\mathrm{Cl}(2)-\mathrm{Mn}(1)-\mathrm{O}(11)-\mathrm{Mn}(3)$ & $-113.93(7)$ \\
\hline $\mathrm{Cl}(2)-\mathrm{Mn}(1)-\mathrm{O}(11)-\mathrm{C}(11)$ & $107.94(18)$ \\
\hline $\mathrm{Cl}(4)-\mathrm{Mn}(1)-\mathrm{O}(11)-\mathrm{Mn}(2)$ & $-135.08(6)$ \\
\hline $\mathrm{Cl}(4)-\mathrm{Mn}(1)-\mathrm{O}(11)-\mathrm{Mn}(3)$ & $123.55(7)$ \\
\hline $\mathrm{Cl}(4)-\mathrm{Mn}(1)-\mathrm{O}(11)-\mathrm{C}(11)$ & $-14.58(19)$ \\
\hline$O(40)-\operatorname{Mn}(1)-O(11)-\operatorname{Mn}(2)$ & $45.5(3)$ \\
\hline$O(40)-\operatorname{Mn}(1)-O(11)-\operatorname{Mn}(3)$ & $-55.9(3)$ \\
\hline $\mathrm{O}(40)-\mathrm{Mn}(1)-\mathrm{O}(11)-\mathrm{C}(11)$ & $166.0(2)$ \\
\hline $\mathrm{Cl}(1)-\mathrm{Mn}(1)-\mathrm{O}(40)-\mathrm{C}(41)$ & $-75.2(2)$ \\
\hline $\mathrm{Cl}(1)-\mathrm{Mn}(1)-\mathrm{O}(40)-\mathrm{C}(44)$ & $68.6(2)$ \\
\hline $\mathrm{Cl}(2)-\mathrm{Mn}(1)-\mathrm{O}(40)-\mathrm{C}(41)$ & $54.4(2)$ \\
\hline $\mathrm{Cl}(2)-\mathrm{Mn}(1)-\mathrm{O}(40)-\mathrm{C}(44)$ & $-161.9(2)$ \\
\hline $\mathrm{Cl}(4)-\mathrm{Mn}(1)-\mathrm{O}(40)-\mathrm{C}(41)$ & $176.8(2)$ \\
\hline $\mathrm{Cl}(4)-\mathrm{Mn}(1)-\mathrm{O}(40)-\mathrm{C}(44)$ & $-39.5(2)$ \\
\hline $\mathrm{O}(11)-\mathrm{Mn}(1)-\mathrm{O}(40)-\mathrm{C}(41)$ & $-3.8(4)$ \\
\hline $\mathrm{O}(11)-\mathrm{Mn}(1)-\mathrm{O}(40)-\mathrm{C}(44)$ & $140.0(3)$ \\
\hline $\mathrm{Cl}(3)-\mathrm{Mn}(2)-\mathrm{Cl}(2)-\mathrm{Mn}(1)$ & $74.66(4)$ \\
\hline $\mathrm{O}(10)-\mathrm{Mn}(2)-\mathrm{Cl}(2)-\mathrm{Mn}(1)$ & $-86.90(6)$ \\
\hline $\mathrm{O}(11)-\mathrm{Mn}(2)-\mathrm{Cl}(2)-\mathrm{Mn}(1)$ & $-10.80(6)$ \\
\hline$O(20)-\operatorname{Mn}(2)-C l(2)-\operatorname{Mn}(1)$ & $-177.31(6)$ \\
\hline $\mathrm{Cl}(2)-\mathrm{Mn}(2)-\mathrm{Cl}(3)-\mathrm{Mn}(3)$ & $-105.18(4)$ \\
\hline$O(11)-\operatorname{Mn}(2)-\mathrm{Cl}(3)-\mathrm{Mn}(3)$ & $-19.77(6)$ \\
\hline$O(20)-\operatorname{Mn}(2)-C l(3)-\operatorname{Mn}(3)$ & $159.82(7)$ \\
\hline $\mathrm{O}(21)-\mathrm{Mn}(2)-\mathrm{Cl}(3)-\mathrm{Mn}(3)$ & $82.24(6)$ \\
\hline $\mathrm{Cl}(2)-\mathrm{Mn}(2)-\mathrm{O}(10)-\mathrm{C}(12)$ & $79.96(17)$ \\
\hline $\mathrm{Cl}(2)-\mathrm{Mn}(2)-\mathrm{O}(10)-\mathrm{C}(13)$ & $-52.9(2)$ \\
\hline $\mathrm{O}(11)-\mathrm{Mn}(2)-\mathrm{O}(10)-\mathrm{C}(12)$ & $-5.83(17)$ \\
\hline $\mathrm{O}(11)-\mathrm{Mn}(2)-\mathrm{O}(10)-\mathrm{C}(13)$ & $-138.7(3)$ \\
\hline$O(20)-\operatorname{Mn}(2)-O(10)-C(12)$ & $173.56(18)$ \\
\hline$O(20)-\operatorname{Mn}(2)-O(10)-C(13)$ & $40.7(2)$ \\
\hline $\mathrm{O}(21)-\mathrm{Mn}(2)-\mathrm{O}(10)-\mathrm{C}(12)$ & $-109.11(18)$ \\
\hline$O(21)-\operatorname{Mn}(2)-O(10)-C(13)$ & $118.0(2)$ \\
\hline $\mathrm{Cl}(2)-\mathrm{Mn}(2)-\mathrm{O}(11)-\mathrm{Mn}(1)$ & $12.58(7)$ \\
\hline $\mathrm{Cl}(2)-\mathrm{Mn}(2)-\mathrm{O}(11)-\mathrm{Mn}(3)$ & $117.02(7)$ \\
\hline $\mathrm{Cl}(2)-\mathrm{Mn}(2)-\mathrm{O}(11)-\mathrm{C}(11)$ & $-113.52(16)$ \\
\hline $\mathrm{Cl}(3)-\mathrm{Mn}(2)-\mathrm{O}(11)-\mathrm{Mn}(1)$ & $-81.53(7)$ \\
\hline $\mathrm{Cl}(3)-\mathrm{Mn}(2)-\mathrm{O}(11)-\mathrm{Mn}(3)$ & $22.91(6)$ \\
\hline $\mathrm{Cl}(3)-\mathrm{Mn}(2)-\mathrm{O}(11)-\mathrm{C}(11)$ & $152.37(17)$ \\
\hline$O(10)-\operatorname{Mn}(2)-O(11)-\operatorname{Mn}(1)$ & $102.79(9)$ \\
\hline$O(10)-\operatorname{Mn}(2)-O(11)-\operatorname{Mn}(3)$ & $-152.78(9)$ \\
\hline $\mathrm{O}(10)-\mathrm{Mn}(2)-\mathrm{O}(11)-\mathrm{C}(11)$ & $-23.32(17)$ \\
\hline$O(21)-\operatorname{Mn}(2)-O(11)-\operatorname{Mn}(1)$ & $-173.32(8)$ \\
\hline$O(21)-\operatorname{Mn}(2)-O(11)-\operatorname{Mn}(3)$ & $-68.88(9)$ \\
\hline
\end{tabular}




\begin{tabular}{|c|c|}
\hline $\mathrm{O}(21)-\mathrm{Mn}(2)-\mathrm{O}(11)-\mathrm{C}(11)$ & $60.57(18)$ \\
\hline $\mathrm{Cl}(2)-\mathrm{Mn}(2)-\mathrm{O}(20)-\mathrm{C}(22)$ & $-177.64(17)$ \\
\hline $\mathrm{Cl}(2)-\mathrm{Mn}(2)-\mathrm{O}(20)-\mathrm{C}(23)$ & $-35.1(2)$ \\
\hline $\mathrm{Cl}(3)-\mathrm{Mn}(2)-\mathrm{O}(20)-\mathrm{C}(22)$ & $-82.52(18)$ \\
\hline $\mathrm{Cl}(3)-\mathrm{Mn}(2)-\mathrm{O}(20)-\mathrm{C}(23)$ & $60.0(2)$ \\
\hline $\mathrm{O}(10)-\mathrm{Mn}(2)-\mathrm{O}(20)-\mathrm{C}(22)$ & $93.17(18)$ \\
\hline$O(10)-M n(2)-O(20)-C(23)$ & $-124.3(2)$ \\
\hline $\mathrm{O}(21)-\mathrm{Mn}(2)-\mathrm{O}(20)-\mathrm{C}(22)$ & $6.22(18)$ \\
\hline $\mathrm{O}(21)-\mathrm{Mn}(2)-\mathrm{O}(20)-\mathrm{C}(23)$ & $148.8(3)$ \\
\hline $\mathrm{Cl}(3)-\mathrm{Mn}(2)-\mathrm{O}(21)-\mathrm{Al}(1)$ & $-61.14(15)$ \\
\hline $\mathrm{Cl}(3)-\mathrm{Mn}(2)-\mathrm{O}(21)-\mathrm{C}(21)$ & $131.41(17)$ \\
\hline$O(10)-M n(2)-O(21)-A I(1)$ & $100.18(16)$ \\
\hline $\mathrm{O}(10)-\mathrm{Mn}(2)-\mathrm{O}(21)-\mathrm{C}(21)$ & $-67.28(18)$ \\
\hline $\mathrm{O}(11)-\mathrm{Mn}(2)-\mathrm{O}(21)-\mathrm{Al}(1)$ & $25.09(17)$ \\
\hline $\mathrm{O}(11)-\mathrm{Mn}(2)-\mathrm{O}(21)-\mathrm{C}(21)$ & $-142.37(17)$ \\
\hline $\mathrm{O}(20)-\mathrm{Mn}(2)-\mathrm{O}(21)-\mathrm{Al}(1)$ & $-168.70(17)$ \\
\hline $\mathrm{O}(20)-\mathrm{Mn}(2)-\mathrm{O}(21)-\mathrm{C}(21)$ & $23.84(17)$ \\
\hline $\mathrm{Cl}(3)-\mathrm{Mn}(3)-\mathrm{Cl}(1)-\mathrm{Mn}(1)$ & $-71.46(4)$ \\
\hline $\mathrm{O}(11)-\mathrm{Mn}(3)-\mathrm{Cl}(1)-\mathrm{Mn}(1)$ & $13.67(6)$ \\
\hline $\mathrm{O}(30)-\mathrm{Mn}(3)-\mathrm{Cl}(1)-\mathrm{Mn}(1)$ & $109.12(6)$ \\
\hline $\mathrm{O}(50)-\mathrm{Mn}(3)-\mathrm{Cl}(1)-\mathrm{Mn}(1)$ & $-166.79(6)$ \\
\hline $\mathrm{Cl}(1)-\mathrm{Mn}(3)-\mathrm{Cl}(3)-\mathrm{Mn}(2)$ & $103.06(3)$ \\
\hline $\mathrm{O}(11)-\mathrm{Mn}(3)-\mathrm{Cl}(3)-\mathrm{Mn}(2)$ & $19.70(6)$ \\
\hline $\mathrm{O}(31)-\mathrm{Mn}(3)-\mathrm{Cl}(3)-\mathrm{Mn}(2)$ & $-73.43(6)$ \\
\hline $\mathrm{O}(50)-\mathrm{Mn}(3)-\mathrm{Cl}(3)-\mathrm{Mn}(2)$ & $-166.59(7)$ \\
\hline $\mathrm{Cl}(1)-\mathrm{Mn}(3)-\mathrm{O}(11)-\mathrm{Mn}(1)$ & $-15.83(6)$ \\
\hline $\mathrm{Cl}(1)-\mathrm{Mn}(3)-\mathrm{O}(11)-\mathrm{Mn}(2)$ & $-119.94(7)$ \\
\hline $\mathrm{Cl}(1)-\mathrm{Mn}(3)-\mathrm{O}(11)-\mathrm{C}(11)$ & $119.76(19)$ \\
\hline $\mathrm{Cl}(3)-\mathrm{Mn}(3)-\mathrm{O}(11)-\mathrm{Mn}(1)$ & $81.10(7)$ \\
\hline $\mathrm{Cl}(3)-\mathrm{Mn}(3)-\mathrm{O}(11)-\mathrm{Mn}(2)$ & $-23.01(6)$ \\
\hline $\mathrm{Cl}(3)-\mathrm{Mn}(3)-\mathrm{O}(11)-\mathrm{C}(11)$ & $-143.31(19)$ \\
\hline $\mathrm{O}(30)-\mathrm{Mn}(3)-\mathrm{O}(11)-\mathrm{Mn}(1)$ & $-108.32(9)$ \\
\hline $\mathrm{O}(30)-\mathrm{Mn}(3)-\mathrm{O}(11)-\mathrm{Mn}(2)$ & $147.58(8)$ \\
\hline $\mathrm{O}(30)-\mathrm{Mn}(3)-\mathrm{O}(11)-\mathrm{C}(11)$ & $27.3(2)$ \\
\hline $\mathrm{O}(31)-\mathrm{Mn}(3)-\mathrm{O}(11)-\mathrm{Mn}(1)$ & $176.21(8)$ \\
\hline $\mathrm{O}(31)-\mathrm{Mn}(3)-\mathrm{O}(11)-\mathrm{Mn}(2)$ & $72.10(9)$ \\
\hline $\mathrm{O}(31)-\mathrm{Mn}(3)-\mathrm{O}(11)-\mathrm{C}(11)$ & $-48.2(2)$ \\
\hline $\mathrm{Cl}(1)-\mathrm{Mn}(3)-\mathrm{O}(30)-\mathrm{C}(32)$ & $177.85(17)$ \\
\hline $\mathrm{Cl}(1)-\mathrm{Mn}(3)-\mathrm{O}(30)-\mathrm{C}(33)$ & $-25.5(3)$ \\
\hline $\mathrm{O}(11)-\mathrm{Mn}(3)-\mathrm{O}(30)-\mathrm{C}(32)$ & $-98.00(18)$ \\
\hline$O(11)-\mathrm{Mn}(3)-\mathrm{O}(30)-\mathrm{C}(33)$ & $58.7(3)$ \\
\hline $\mathrm{O}(31)-\mathrm{Mn}(3)-\mathrm{O}(30)-\mathrm{C}(32)$ & $-5.89(18)$ \\
\hline $\mathrm{O}(31)-\mathrm{Mn}(3)-\mathrm{O}(30)-\mathrm{C}(33)$ & $150.8(3)$ \\
\hline $\mathrm{O}(50)-\mathrm{Mn}(3)-\mathrm{O}(30)-\mathrm{C}(32)$ & $88.42(19)$ \\
\hline $\mathrm{O}(50)-\mathrm{Mn}(3)-\mathrm{O}(30)-\mathrm{C}(33)$ & $-114.9(3)$ \\
\hline $\mathrm{Cl}(3)-\mathrm{Mn}(3)-\mathrm{O}(31)-\mathrm{Al}(1)$ & $39.90(17)$ \\
\hline $\mathrm{Cl}(3)-\mathrm{Mn}(3)-\mathrm{O}(31)-\mathrm{C}(31)$ & $-145.81(16)$ \\
\hline $\mathrm{O}(11)-\mathrm{Mn}(3)-\mathrm{O}(31)-\mathrm{Al}(1)$ & $-46.28(18)$ \\
\hline $\mathrm{O}(11)-\mathrm{Mn}(3)-\mathrm{O}(31)-\mathrm{C}(31)$ & $128.01(17)$ \\
\hline $\mathrm{O}(30)-\mathrm{Mn}(3)-\mathrm{O}(31)-\mathrm{Al}(1)$ & $-141.30(19)$ \\
\hline $\mathrm{O}(30)-\mathrm{Mn}(3)-\mathrm{O}(31)-\mathrm{C}(31)$ & $32.99(17)$ \\
\hline $\mathrm{O}(50)-\mathrm{Mn}(3)-\mathrm{O}(31)-\mathrm{Al}(1)$ & $135.50(18)$ \\
\hline $\mathrm{O}(50)-\mathrm{Mn}(3)-\mathrm{O}(31)-\mathrm{C}(31)$ & $-50.21(18)$ \\
\hline $\mathrm{Cl}(1)-\mathrm{Mn}(3)-\mathrm{O}(50)-\mathrm{C}(51)$ & $125.1(2)$ \\
\hline $\mathrm{Cl}(1)-\mathrm{Mn}(3)-\mathrm{O}(50)-\mathrm{C}(54)$ & $-52.2(2)$ \\
\hline $\mathrm{Cl}(3)-\mathrm{Mn}(3)-\mathrm{O}(50)-\mathrm{C}(51)$ & $28.7(2)$ \\
\hline $\mathrm{Cl}(3)-\mathrm{Mn}(3)-\mathrm{O}(50)-\mathrm{C}(54)$ & $-148.6(2)$ \\
\hline $\mathrm{O}(30)-\mathrm{Mn}(3)-\mathrm{O}(50)-\mathrm{C}(51)$ & $-141.8(2)$ \\
\hline$O(30)-\mathrm{Mn}(3)-\mathrm{O}(50)-\mathrm{C}(54)$ & $41.0(2)$ \\
\hline $\mathrm{O}(31)-\mathrm{Mn}(3)-\mathrm{O}(50)-\mathrm{C}(51)$ & $-66.9(2)$ \\
\hline $\mathrm{O}(31)-\mathrm{Mn}(3)-\mathrm{O}(50)-\mathrm{C}(54)$ & $115.8(2)$ \\
\hline
\end{tabular}




\begin{tabular}{|c|c|}
\hline$O(31)-A l(1)-O(21)-\operatorname{Mn}(2)$ & $10.62(18)$ \\
\hline$O(31)-A 1(1)-O(21)-C(21)$ & $177.1(2)$ \\
\hline $\mathrm{C}(1)-\mathrm{Al}(1)-\mathrm{O}(21)-\mathrm{Mn}(2)$ & $-105.85(19)$ \\
\hline$C(1)-A 1(1)-O(21)-C(21)$ & $60.7(2)$ \\
\hline $\mathrm{C}(2)-\mathrm{Al}(1)-\mathrm{O}(21)-\mathrm{Mn}(2)$ & $129.23(17)$ \\
\hline$C(2)-A I(1)-O(21)-C(21)$ & $-64.3(2)$ \\
\hline$O(21)-A I(1)-O(31)-\operatorname{Mn}(3)$ & $2.0(2)$ \\
\hline$O(21)-A I(1)-O(31)-C(31)$ & $-172.01(19)$ \\
\hline $\mathrm{C}(1)-\mathrm{Al}(1)-\mathrm{O}(31)-\mathrm{Mn}(3)$ & $118.76(19)$ \\
\hline$C(1)-A 1(1)-O(31)-C(31)$ & $-55.2(2)$ \\
\hline $\mathrm{C}(2)-\mathrm{Al}(1)-\mathrm{O}(31)-\mathrm{Mn}(3)$ & $-116.50(19)$ \\
\hline$C(2)-A I(1)-O(31)-C(31)$ & $69.5(2)$ \\
\hline $\operatorname{Mn}(2)-O(10)-C(12)-C(11)$ & $32.1(3)$ \\
\hline$C(13)-O(10)-C(12)-C(11)$ & $169.5(2)$ \\
\hline $\operatorname{Mn}(2)-\mathrm{O}(11)-\mathrm{C}(11)-\mathrm{C}(12)$ & $50.0(2)$ \\
\hline $\operatorname{Mn}(1)-O(11)-C(11)-C(12)$ & $-66.2(3)$ \\
\hline $\operatorname{Mn}(3)-\mathrm{O}(11)-\mathrm{C}(11)-\mathrm{C}(12)$ & $164.66(17)$ \\
\hline$C(23)-O(20)-C(22)-C(21)$ & $178.9(3)$ \\
\hline $\operatorname{Mn}(2)-O(20)-C(22)-C(21)$ & $-33.3(3)$ \\
\hline $\mathrm{Al}(1)-\mathrm{O}(21)-\mathrm{C}(21)-\mathrm{C}(22)$ & $139.7(2)$ \\
\hline $\operatorname{Mn}(2)-O(21)-C(21)-C(22)$ & $-50.8(2)$ \\
\hline$C(33)-O(30)-C(32)-C(31)$ & $178.1(3)$ \\
\hline $\operatorname{Mn}(3)-O(30)-C(32)-C(31)$ & $-21.1(3)$ \\
\hline $\operatorname{Mn}(3)-O(31)-C(31)-C(32)$ & $-56.9(2)$ \\
\hline $\mathrm{Al}(1)-\mathrm{O}(31)-\mathrm{C}(31)-\mathrm{C}(32)$ & $118.8(2)$ \\
\hline $\operatorname{Mn}(1)-\mathrm{O}(40)-\mathrm{C}(41)-\mathrm{C}(42)$ & $168.7(2)$ \\
\hline$C(41)-O(40)-C(44)-C(43)$ & $0.0(4)$ \\
\hline $\operatorname{Mn}(1)-O(40)-C(44)-C(43)$ & $-148.4(3)$ \\
\hline$C(44)-O(40)-C(41)-C(42)$ & $21.3(3)$ \\
\hline $\operatorname{Mn}(3)-O(50)-C(54)-C(53)$ & $156.8(2)$ \\
\hline$C(54)-O(50)-C(51)-C(52)$ & $-3.4(3)$ \\
\hline $\operatorname{Mn}(3)-O(50)-C(51)-C(52)$ & $178.96(18)$ \\
\hline$C(51)-O(50)-C(54)-C(53)$ & $-20.8(4)$ \\
\hline $\mathrm{O}(11)-\mathrm{C}(11)-\mathrm{C}(12)-\mathrm{O}(10)$ & $-54.7(3)$ \\
\hline $\mathrm{O}(21)-\mathrm{C}(21)-\mathrm{C}(22)-\mathrm{O}(20)$ & $56.0(3)$ \\
\hline $\mathrm{O}(31)-\mathrm{C}(31)-\mathrm{C}(32)-\mathrm{O}(30)$ & $51.2(3)$ \\
\hline$O(40)-C(41)-C(42)-C(43)$ & $-34.3(3)$ \\
\hline$C(41)-C(42)-C(43)-C(44)$ & $33.8(4)$ \\
\hline$C(42)-C(43)-C(44)-O(40)$ & $-21 \cdot 4(4)$ \\
\hline$O(50)-C(51)-C(52)-C(53)$ & $25.6(3)$ \\
\hline$C(51)-C(52)-C(53)-C(54)$ & $-37.6(3)$ \\
\hline$C(52)-C(53)-C(54)-O(50)$ & $36.0(3)$ \\
\hline
\end{tabular}




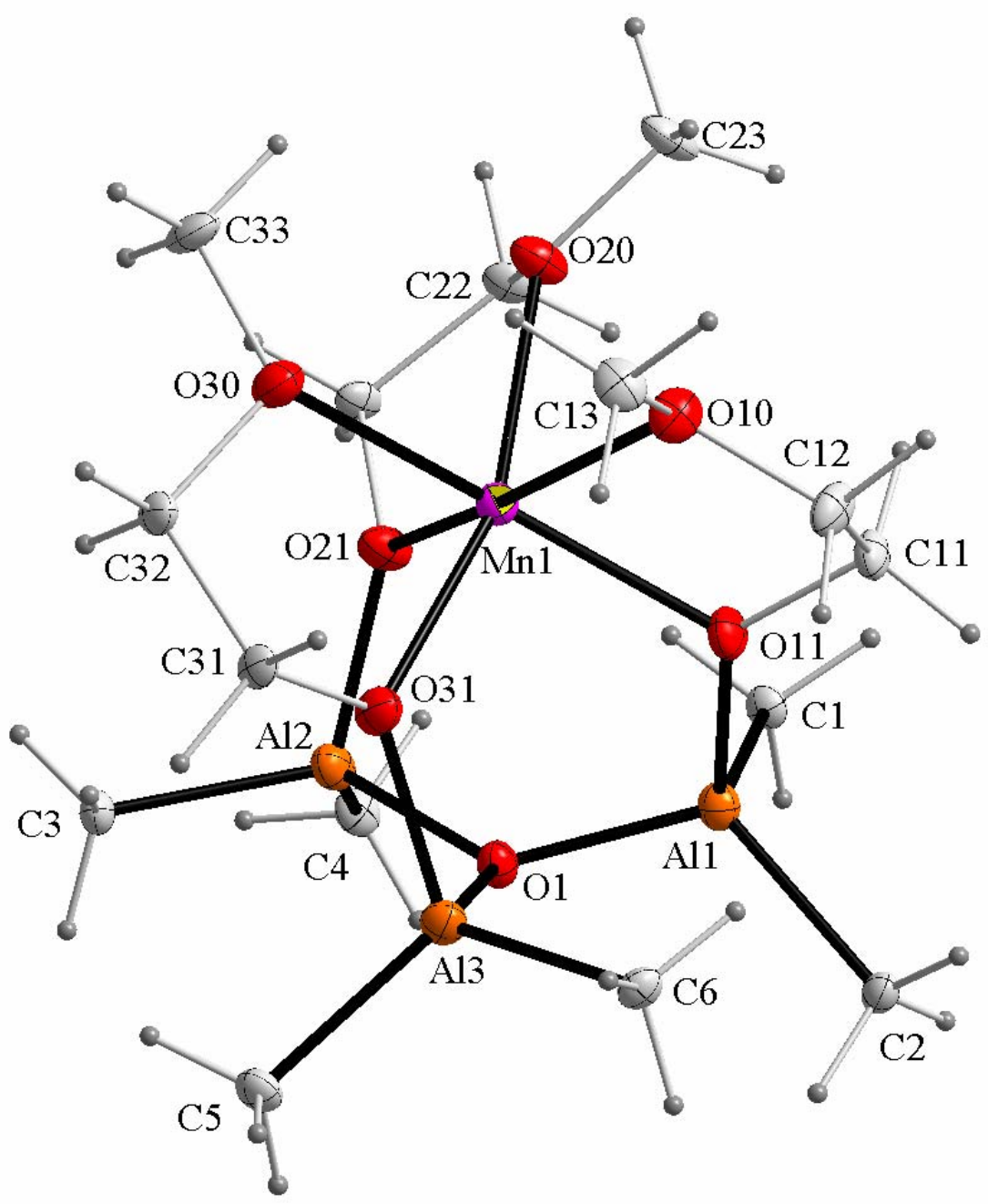

Figure S2. Molecular structure of the $\left[\mathrm{MnAl}_{3}\left(\mu_{3}-\mathrm{O}\right)\left(\mu-\mathrm{OCH}_{2} \mathrm{CH}_{2} \mathrm{OCH}_{3}\right)_{3}(\mathrm{Me})_{6}\right]$

(ORTEP plot, thermal ellipsoids set at $50 \%$ ). 
Table S21. Crystal data and structure refinement for $\left[\mathrm{MnAl}_{3}\left(\mu_{3}-\mathrm{O}\right)\left(\mu-\mathrm{OCH}_{2} \mathrm{CH}_{2} \mathrm{OCH}_{3}\right)_{3}(\mathrm{Me})_{6}\right]$ (3).

Compound

Chemical formula

Formula weight

$\lambda(\AA)$

$\mathrm{T}(\mathrm{K})$

Space group

Crystal system

Unit cell dimensions

a $(\AA)$

$\mathrm{b}(\AA)$

$c(\AA)$

alpha $\left({ }^{\circ}\right)$

beta $\left({ }^{\circ}\right)$

gamma $\left({ }^{\circ}\right)$

$\mathrm{V}\left(\AA^{3}\right)$

Z

$\mathrm{D}_{\mathrm{C}}\left(\mathrm{Mg} / \mathrm{m}^{3}\right)$

$\mathrm{F}(000)$

Habit

Crystal size (mm)

$\mu\left(\mathrm{mm}^{-1}\right)$

Absorption correction

Max. and min.

Type of diffractometer

Diffraction geometry

Theta range $\left({ }^{\circ}\right)$

Number of reflections measured

Number of unique reflections

$\mathrm{R}_{\text {(int) }}$

Number of observed reflections

Refinement method

Number of parameters

Final R1 indices [I>2 $\sigma(I)]$

Final R1 indices (all data)

Goodness-of-fit (S)

Largest diff. peak and hole e. $\AA^{-3}$

\section{CCDC299462}

$\mathrm{C}_{19} \mathrm{H}_{43} \mathrm{AlCl}_{4} \mathrm{Mn}_{3} \mathrm{O}_{8}$

733.13

0.71073

$100(2)$

$\mathrm{P} 21 / \mathrm{n}$

Monoclinic

$$
\begin{aligned}
& 14.271(4) \\
& 15.302(5) \\
& 14.920(5) \\
& 90 \\
& 91.69(2) \\
& 90
\end{aligned}
$$

$3256.7(18)$

4

1.495

1508

block

$0.293 \times 0.264 \times 0.200$

1.536

Analytical

transmission 0.910 and 0.687

Kuma KM4CCD kappa-axis diffractometer $\omega$

2.99 to 27.00

21678

7023

0.0541

$5119[\mathrm{I}>2 \sigma(\mathrm{I})]$

least-squares on $\mathrm{F}^{2}$

321

$\mathrm{R}_{1}=0.0442, \mathrm{wR}_{2}=0.0968$

$\mathrm{R}_{1}=0.0680, \mathrm{wR}_{2}=0.1027$

1.012

0.670 and -0.668 
Table S22. Bond lengths [A] and angles [deg] for $\left[\mathrm{MnAl}_{3}\left(\mu_{3}-\mathrm{O}\right)\left(\mu-\mathrm{OCH}_{2} \mathrm{CH}_{2} \mathrm{OCH}_{3}\right)_{3}(\mathrm{Me})_{6}\right]$ (3).

\begin{tabular}{|c|c|}
\hline $\operatorname{Mn}(1)-0(31)$ & $2.111(2)$ \\
\hline $\operatorname{Mn}(1)-O(11)$ & $2.117(2)$ \\
\hline $\operatorname{Mn}(1)-O(21)$ & $2.119(2)$ \\
\hline $\operatorname{Mn}(1)-O(20)$ & $2.242(2)$ \\
\hline $\operatorname{Mn}(1)-O(10)$ & $2.258(2)$ \\
\hline $\operatorname{Mn}(1)-0(30)$ & $2.257(2)$ \\
\hline$A l(1)-O(11)$ & $1.811(2)$ \\
\hline $\mathrm{Al}(1)-\mathrm{O}(1)$ & $1.828(2)$ \\
\hline$A 1(1)-C(1)$ & $1.974(2)$ \\
\hline$A 1(1)-C(2)$ & $1.982(2)$ \\
\hline $\mathrm{Al}(2)-\mathrm{O}(21)$ & $1.815(2)$ \\
\hline $\mathrm{Al}(2)-\mathrm{O}(1)$ & $1.830(2)$ \\
\hline$A l(2)-C(4)$ & $1.976(2)$ \\
\hline$A \perp(2)-C(3)$ & $1.978(2)$ \\
\hline$A I(3)-O(31)$ & $1.815(2)$ \\
\hline$A l(3)-O(1)$ & $1.829(2)$ \\
\hline$A \perp(3)-C(5)$ & $1.972(2)$ \\
\hline$A l(3)-C(6)$ & $1.978(2)$ \\
\hline$O(10)-C(13)$ & $1.434(2)$ \\
\hline$O(10)-C(12)$ & $1.441(2)$ \\
\hline$O(11)-C(11)$ & $1.416(2)$ \\
\hline$C(11)-C(12)$ & $1.506(3)$ \\
\hline$O(20)-C(23)$ & $1.429(2)$ \\
\hline$O(20)-C(22)$ & $1.441(2)$ \\
\hline $\mathrm{O}(21)-\mathrm{C}(21)$ & $1.421(2)$ \\
\hline$C(21)-C(22)$ & $1.509(3)$ \\
\hline$O(30)-C(33)$ & $1.435(2)$ \\
\hline$O(30)-C(32)$ & $1.439(2)$ \\
\hline $\mathrm{O}(31)-\mathrm{C}(31)$ & $1.420(2)$ \\
\hline$C(31)-C(32)$ & $1.510(3)$ \\
\hline $\mathrm{O}(31)-\mathrm{Mn}(1)-\mathrm{O}(11)$ & $95.46(5)$ \\
\hline $\mathrm{O}(31)-\mathrm{Mn}(1)-\mathrm{O}(21)$ & $95.62(5)$ \\
\hline$O(11)-\mathrm{Mn}(1)-\mathrm{O}(21)$ & $96.11(5)$ \\
\hline$O(31)-\operatorname{Mn}(1)-O(20)$ & $162.19(5)$ \\
\hline $\mathrm{O}(11)-\mathrm{Mn}(1)-\mathrm{O}(20)$ & $100.93(5)$ \\
\hline$O(21)-\operatorname{Mn}(1)-O(20)$ & $75.90(5)$ \\
\hline$O(31)-\operatorname{Mn}(1)-O(10)$ & $100.48(5)$ \\
\hline$O(11)-\operatorname{Mn}(1)-O(10)$ & $75.84(5)$ \\
\hline$O(21)-\operatorname{Mn}(1)-O(10)$ & $162.57(5)$ \\
\hline$O(20)-\operatorname{Mn}(1)-O(10)$ & $90.29(5)$ \\
\hline$O(31)-\operatorname{Mn}(1)-O(30)$ & $76.04(5)$ \\
\hline$O(11)-\mathrm{Mn}(1)-\mathrm{O}(30)$ & $161.51(5)$ \\
\hline$O(21)-\operatorname{Mn}(1)-O(30)$ & $101.00(5)$ \\
\hline$O(20)-\operatorname{Mn}(1)-O(30)$ & $90.06(5)$ \\
\hline$O(10)-M n(1)-O(30)$ & $89.41(5)$ \\
\hline$O(11)-A I(1)-O(1)$ & $96.03(6)$ \\
\hline$O(11)-A 1(1)-C(1)$ & $106.82(7)$ \\
\hline $\mathrm{O}(1)-\mathrm{Al}(1)-\mathrm{C}(1)$ & $116.22(7)$ \\
\hline$O(11)-A 1(1)-C(2)$ & $113.29(7)$ \\
\hline$O(1)-A I(1)-C(2)$ & $112.93(7)$ \\
\hline $\mathrm{C}(1)-\mathrm{Al}(1)-\mathrm{C}(2)$ & $110.65(8)$ \\
\hline$O(21)-A 1(2)-O(1)$ & $96.05(6)$ \\
\hline$O(21)-A 1(2)-C(4)$ & $112.00(7)$ \\
\hline$O(1)-A I(2)-C(4)$ & $114.39(8)$ \\
\hline$O(21)-A I(2)-C(3)$ & $108.19(7)$ \\
\hline
\end{tabular}




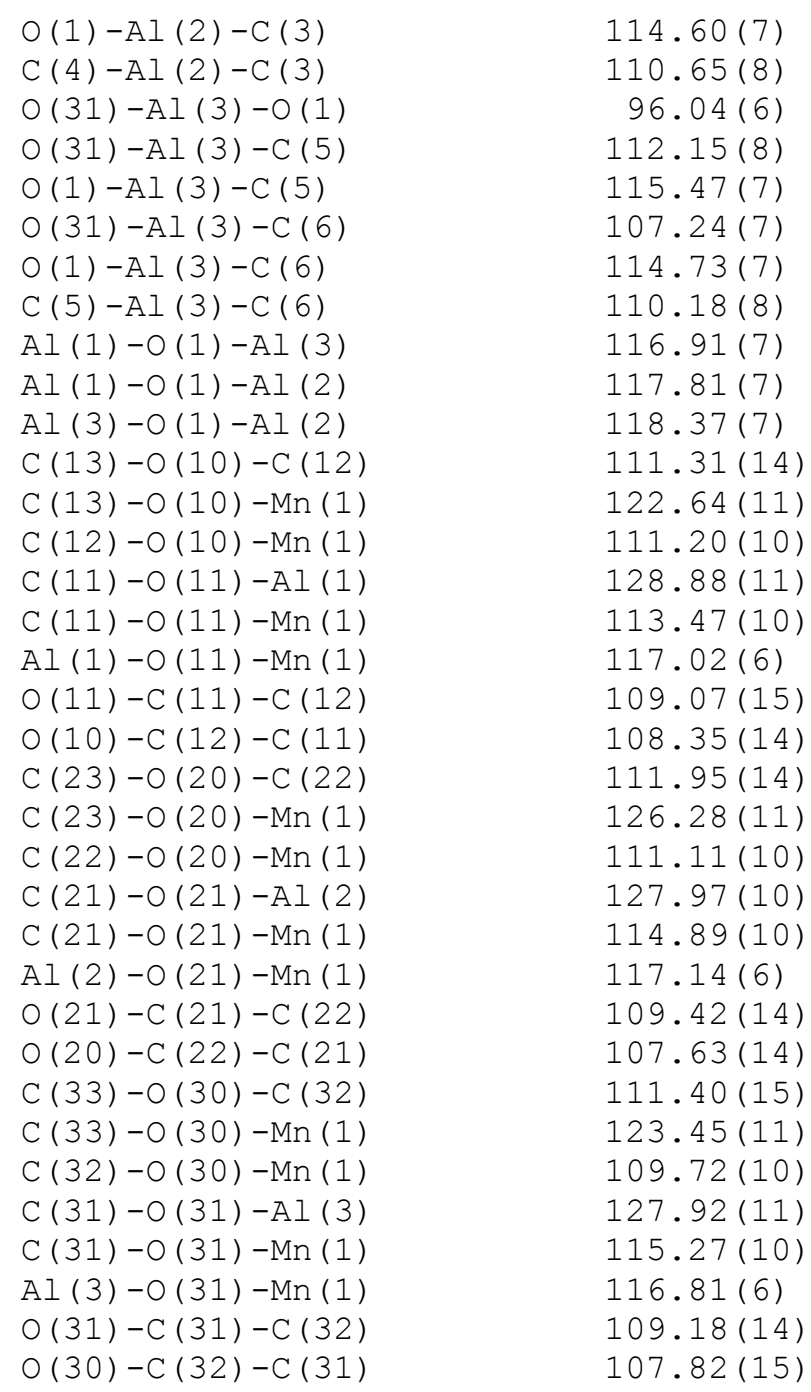


Table S23. Torsion angles [deg] for $\left[\mathrm{MnAl}_{3}\left(\mu_{3}-\mathrm{O}\right)\left(\mu-\mathrm{OCH}_{2} \mathrm{CH}_{2} \mathrm{OCH}_{3}\right)_{3}(\mathrm{Me})_{6}\right]$ (3).

\begin{tabular}{|c|c|}
\hline$O(11)-A I(1)-O(1)-A I(3)$ & $-57.90(8)$ \\
\hline $\mathrm{C}(1)-\mathrm{Al}(1)-\mathrm{O}(1)-\mathrm{Al}(3)$ & $-170.01(8)$ \\
\hline$C(2)-A I(1)-O(1)-A I(3)$ & $60.56(10)$ \\
\hline$O(11)-A I(1)-O(1)-A I(2)$ & $92.58(8)$ \\
\hline$C(1)-A l(1)-O(1)-A l(2)$ & $-19.54(10)$ \\
\hline $\mathrm{C}(2)-\mathrm{Al}(1)-\mathrm{O}(1)-\mathrm{Al}(2)$ & $-148.97(8)$ \\
\hline$O(31)-A I(3)-O(1)-A I(1)$ & $92.81(8)$ \\
\hline$C(5)-A l(3)-O(1)-A l(1)$ & $-149.14(8)$ \\
\hline $\mathrm{C}(6)-\mathrm{Al}(3)-\mathrm{O}(1)-\mathrm{Al}(1)$ & $-19.33(10)$ \\
\hline$O(31)-A I(3)-O(1)-A l(2)$ & $-57.50(8)$ \\
\hline$C(5)-A l(3)-O(1)-A l(2)$ & $60.56(10)$ \\
\hline $\mathrm{C}(6)-\mathrm{Al}(3)-\mathrm{O}(1)-\mathrm{Al}(2)$ & $-169.64(8)$ \\
\hline$O(21)-\mathrm{Al}(2)-\mathrm{O}(1)-\mathrm{Al}(1)$ & $-58.61(8)$ \\
\hline $\mathrm{C}(4)-\mathrm{Al}(2)-\mathrm{O}(1)-\mathrm{Al}(1)$ & $58.89(10)$ \\
\hline $\mathrm{C}(3)-\mathrm{Al}(2)-\mathrm{O}(1)-\mathrm{Al}(1)$ & $-171.80(8)$ \\
\hline$O(21)-A I(2)-O(1)-A I(3)$ & $91.42(8)$ \\
\hline $\mathrm{C}(4)-\mathrm{Al}(2)-\mathrm{O}(1)-\mathrm{Al}(3)$ & $-151.08(8)$ \\
\hline$C(3)-A I(2)-O(1)-A I(3)$ & $-21.77(10)$ \\
\hline $\mathrm{O}(31)-\mathrm{Mn}(1)-\mathrm{O}(10)-\mathrm{C}(13)$ & $-50.01(14)$ \\
\hline$O(11)-\mathrm{Mn}(1)-\mathrm{O}(10)-\mathrm{C}(13)$ & $-143.06(14)$ \\
\hline $\mathrm{O}(21)-\mathrm{Mn}(1)-\mathrm{O}(10)-\mathrm{C}(13)$ & $152.84(16)$ \\
\hline $\mathrm{O}(20)-\mathrm{Mn}(1)-\mathrm{O}(10)-\mathrm{C}(13)$ & $115.73(13)$ \\
\hline$O(30)-\mathrm{Mn}(1)-\mathrm{O}(10)-\mathrm{C}(13)$ & $25.67(13)$ \\
\hline $\mathrm{O}(31)-\mathrm{Mn}(1)-\mathrm{O}(10)-\mathrm{C}(12)$ & $85.33(12)$ \\
\hline $\mathrm{O}(11)-\mathrm{Mn}(1)-\mathrm{O}(10)-\mathrm{C}(12)$ & $-7.72(12)$ \\
\hline $\mathrm{O}(21)-\mathrm{Mn}(1)-\mathrm{O}(10)-\mathrm{C}(12)$ & $-71.8(2)$ \\
\hline $\mathrm{O}(20)-\mathrm{Mn}(1)-\mathrm{O}(10)-\mathrm{C}(12)$ & $-108.92(12)$ \\
\hline$O(30)-\mathrm{Mn}(1)-\mathrm{O}(10)-\mathrm{C}(12)$ & $161.02(12)$ \\
\hline $\mathrm{O}(1)-\mathrm{A} I(1)-\mathrm{O}(11)-\mathrm{C}(11)$ & $161.01(14)$ \\
\hline$C(1)-A 1(1)-O(11)-C(11)$ & $-79.25(16)$ \\
\hline $\mathrm{C}(2)-\mathrm{Al}(1)-\mathrm{O}(11)-\mathrm{C}(11)$ & $42.84(16)$ \\
\hline $\mathrm{O}(1)-\mathrm{Al}(1)-\mathrm{O}(11)-\mathrm{Mn}(1)$ & $-28.79(7)$ \\
\hline $\mathrm{C}(1)-\mathrm{Al}(1)-\mathrm{O}(11)-\mathrm{Mn}(1)$ & $90.96(9)$ \\
\hline $\mathrm{C}(2)-\mathrm{Al}(1)-\mathrm{O}(11)-\mathrm{Mn}(1)$ & $-146.96(7)$ \\
\hline $\mathrm{O}(31)-\mathrm{Mn}(1)-\mathrm{O}(11)-\mathrm{C}(11)$ & $-120.64(12)$ \\
\hline $\mathrm{O}(21)-\mathrm{Mn}(1)-\mathrm{O}(11)-\mathrm{C}(11)$ & $143.10(11)$ \\
\hline $\mathrm{O}(20)-\mathrm{Mn}(1)-\mathrm{O}(11)-\mathrm{C}(11)$ & $66.33(12)$ \\
\hline $\mathrm{O}(10)-\mathrm{Mn}(1)-\mathrm{O}(11)-\mathrm{C}(11)$ & $-21.18(11)$ \\
\hline $\mathrm{O}(30)-\mathrm{Mn}(1)-\mathrm{O}(11)-\mathrm{C}(11)$ & $-59.2(2)$ \\
\hline $\mathrm{O}(31)-\mathrm{Mn}(1)-\mathrm{O}(11)-\mathrm{Al}(1)$ & $67.66(7)$ \\
\hline$O(21)-\mathrm{Mn}(1)-\mathrm{O}(11)-\mathrm{Al}(1)$ & $-28.60(7)$ \\
\hline$O(20)-\operatorname{Mn}(1)-O(11)-A 1(1)$ & $-105.37(7)$ \\
\hline $\mathrm{O}(10)-\mathrm{Mn}(1)-\mathrm{O}(11)-\mathrm{Al}(1)$ & $167.12(7)$ \\
\hline$O(30)-\operatorname{Mn}(1)-O(11)-A l(1)$ & $129.09(13)$ \\
\hline $\mathrm{Al}(1)-\mathrm{O}(11)-\mathrm{C}(11)-\mathrm{C}(12)$ & $-143.50(13)$ \\
\hline $\operatorname{Mn}(1)-O(11)-C(11)-C(12)$ & $46.01(18)$ \\
\hline$C(13)-O(10)-C(12)-C(11)$ & $173.37(15)$ \\
\hline $\operatorname{Mn}(1)-\mathrm{O}(10)-\mathrm{C}(12)-\mathrm{C}(11)$ & $32.80(18)$ \\
\hline $\mathrm{O}(11)-\mathrm{C}(11)-\mathrm{C}(12)-\mathrm{O}(10)$ & $-51.4(2)$ \\
\hline $\mathrm{O}(31)-\mathrm{Mn}(1)-\mathrm{O}(20)-\mathrm{C}(23)$ & $141.13(18)$ \\
\hline $\mathrm{O}(11)-\mathrm{Mn}(1)-\mathrm{O}(20)-\mathrm{C}(23)$ & $-62.14(16)$ \\
\hline$O(21)-\mathrm{Mn}(1)-\mathrm{O}(20)-\mathrm{C}(23)$ & $-155.77(16)$ \\
\hline $\mathrm{O}(10)-\mathrm{Mn}(1)-\mathrm{O}(20)-\mathrm{C}(23)$ & $13.49(15)$ \\
\hline$O(30)-\mathrm{Mn}(1)-\mathrm{O}(20)-\mathrm{C}(23)$ & $102.90(16)$ \\
\hline $\mathrm{O}(31)-\mathrm{Mn}(1)-\mathrm{O}(20)-\mathrm{C}(22)$ & $-77.57(19)$ \\
\hline $\mathrm{O}(11)-\mathrm{Mn}(1)-\mathrm{O}(20)-\mathrm{C}(22)$ & $79.16(12)$ \\
\hline
\end{tabular}


$-52.8(2)$

$72.93(12)$

$\mathrm{O}(31)-\mathrm{Mn}(1)-\mathrm{O}(21)-\mathrm{Al}(2)$

$-29.79(8)$

$66.34(8)$

$\mathrm{O}(11)-\mathrm{Mn}(1)-\mathrm{O}(21)-\mathrm{Al}(2)$

$166.11(8)$

$O(20)-\operatorname{Mn}(1)-O(21)-A 1(2)$

$127.64(14)$

(10) $-\mathrm{Mn}(1)-\mathrm{O}(21)-\mathrm{A} I(2)$

$-106.62(7)$

$\mathrm{Al}(2)-\mathrm{O}(21)-\mathrm{C}(21)-\mathrm{C}(22)$

$-140.76(13)$

$39.74(17)$

$\mathrm{Mn}(1)-\mathrm{O}(21)-\mathrm{C}(21)-\mathrm{C}(22)$

$-174.63(16)$

$38.29(16)$

$\mathrm{Mn}(1)-\mathrm{O}(20)-\mathrm{C}(22)-\mathrm{C}(21)$

$-50.84(19)$

$O(21)-C(21)-C(22)-O(20)$

$-150.93(15)$

$144.80(17)$

$-57.81(15)$

$17.83(15)$

$\mathrm{O}(21)-\mathrm{Mn}(1)-\mathrm{O}(30)-\mathrm{C}(33)$

$17.83(15)$

$\mathrm{O}(10)-\mathrm{Mn}(1)-\mathrm{O}(30)-\mathrm{C}(33)$

$-16.40(11)$

$\mathrm{O}(31)-\mathrm{Mn}(1)-\mathrm{O}(30)-\mathrm{C}(32)$

$-80.67(19)$

$76.72(12)$

$\mathrm{O}(21)-\mathrm{Mn}(1)-\mathrm{O}(30)-\mathrm{C}(32)$

$52.36(11)$

$\mathrm{O}(20)-\mathrm{Mn}(1)-\mathrm{O}(30)-\mathrm{C}(32)$

$-117.36(11)$

$151.47(14)$

$30.81(16)$

$\mathrm{O}(1)-\mathrm{Al}(3)-\mathrm{O}(31)-\mathrm{C}(31)$

$\mathrm{C}(5)-\mathrm{Al}(3)-\mathrm{O}(31)-\mathrm{C}(31)$

$\mathrm{C}(6)-\mathrm{Al}(3)-\mathrm{O}(31)-\mathrm{C}(31)$

$-90.27(15)$

$\mathrm{O}(1)-\mathrm{Al}(3)-\mathrm{O}(31)-\mathrm{Mn}(1)$

$-29.40(8)$

$\mathrm{C}(5)-\mathrm{Al}(3)-\mathrm{O}(31)-\mathrm{Mn}(1)$

$\mathrm{C}(6)-\mathrm{Al}(3)-\mathrm{O}(31)-\mathrm{Mn}(1)$

$-150.07(8)$

$88.85(9)$

$\mathrm{O}(11)-\mathrm{Mn}(1)-\mathrm{O}(31)-\mathrm{C}(31)$

$150.52(11)$

$\mathrm{O}(21)-\mathrm{Mn}(1)-\mathrm{O}(31)-\mathrm{C}(31)$

$-112.77(12)$

$-52.4(2)$

$73.94(12)$

$-12.80(11)$

$-28.72(7)$

$67.99(8)$

$128.35(13)$

$-105.29(7)$

$167.96(8)$

$-142.04(13)$

$38.82(18)$

$-179.27(15)$

$40.43(16)$

$-52.08(19)$ 